\title{
Inducible Nitric Oxide Synthase Expression in Brain Injury Cases
}

\author{
Bander Khalid Al-rais* \\ Laboratory specialist, Saudi Arabia. \\ *Corresponding author: Bander Khalid al-rais, Master of forensic and toxicology science PhD in health management, Laboratory \\ specialist, Ministry of health in Saudi Arabia.
}

To Cite This Article: Bander Khalid Al-rais, Inducible Nitric Oxide Synthase Expression in Brain Injury Cases. 2020 - 7(3). AJBSR.MS.ID.001150.

DOI: 10.34297/AJBSR.2020.07.001150.

Received: 畊 January 28, 2020; Published: 㘹 February 18, 2020

\begin{abstract}
Background: The activity of Inducible Nitric Oxide Synthase (iNOS) is increased as a response to several insults to central nervous system causing brain injury.

Objectives: To investigate iNOS expression in cadavers who died as result of traumatic brain injury (TBI), brain hemorrhage and brain congestion

Methodology: Indirect immunohistochemistry was performed on 80 cases of brain injury (autopsies), as well 23 normal brain cases as a control group. The cases were stained for iNOS and interpreted in terms of staining intensity from 0 to indicate negative, 1 weak, 2 moderate and 3 to indicate strong staining reactions.

Results: personal variables (age, sex, brain weight) were not associated significantly with brain injury type. The iNOS was not express in control group. But, strong activity of iNOS was expressed in neurons astrocytes and in cases of TBI and brain hemorrhage. The activity of iNOS in blood vessels was moderate. The rate of expression of iNOS in congestion cases was low in comparison with TBI and hemorrhage cases.

Conclusion: The activity of iNOS was shown to be significantly correlated with TBI and hemorrhage. Strong reactions were localized within neurons and astrocytes. This possibly explains the mediated effects of iNOS on brain blood vessels in terms of blood vessels dilatation to accommodate with the severity of brain injury cases.
\end{abstract}

Keywords: Inducible Nitric Oxide Synthase, Traumatic Brain Injury, Central Nervous System, Inflammatory disease

\section{Introduction}

Nitric oxide synthase contributes through its product nitric oxide to transmission from one neuron to another, to the immune system and to dilating blood vessels. This enzyme has three isoforms, two are constitutive and the third is inducible. The Inducible Nitric Oxide Synthase (iNOS) is not detectable in normal brain condition [1]. It is an inflammatory product that is induced both in secondary damage [2] and in neurological recovery [3]. The inflammation response includes cytokine production, leucocytes infiltrations and microglial activation. A study reported that iNOS is not found in brain until 6-12 hours after Traumatic Brain Injury (TBI) [4].

The said enzyme is expressed in many different cells like epithelial cells, endothelial cells, macrophages and neurons [5]. A paper documented that iNOS has been detected in the vasculature, infiltrating macrophages and tumor cell of human brain [6]. While some researches demonstrated iNOS expression in primary astrocytes upon inflammatory induction as well as in neurological diseases whereas increase expression of iNOS sepsis, asthma, rheumatoid arthritis, atherosclerosis, tuberculosis and multiple sclerosis [7-16]. Hence it is clear that the activity of iNOS is increase as a result of several processes in Central Nervous System (CNS), causing brain injury.

Besides, the known how about the response to the injury is important for practical management of disease. Moreover, iNOS expression in the study population having different environment, nutrition and lifestyle has not been expressed in the study 
population having different environment, nutrition and lifestyle has been expressed particularly in the brain injury cases. Hence in the present attempt iNOS was investigated in the brain cadavers who died as result of traumatic brain injury, brain hemorrhage and brain congestion taken together (Figure 1-3), these observations suggest a relevant role of iNOS and its product NO in the inflammatory disease.

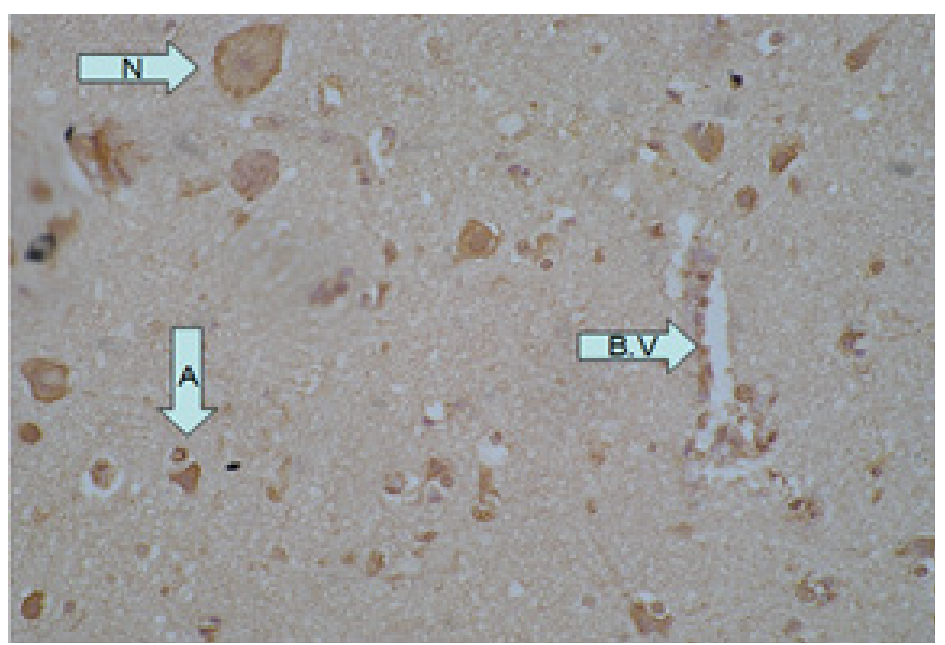

Figure 1: Shows strong expression of iNOS in TBI case (+3 staining intensity in astrocytes (A), neurons (N) and blood vessels (B.V) as indicated by the arrow).

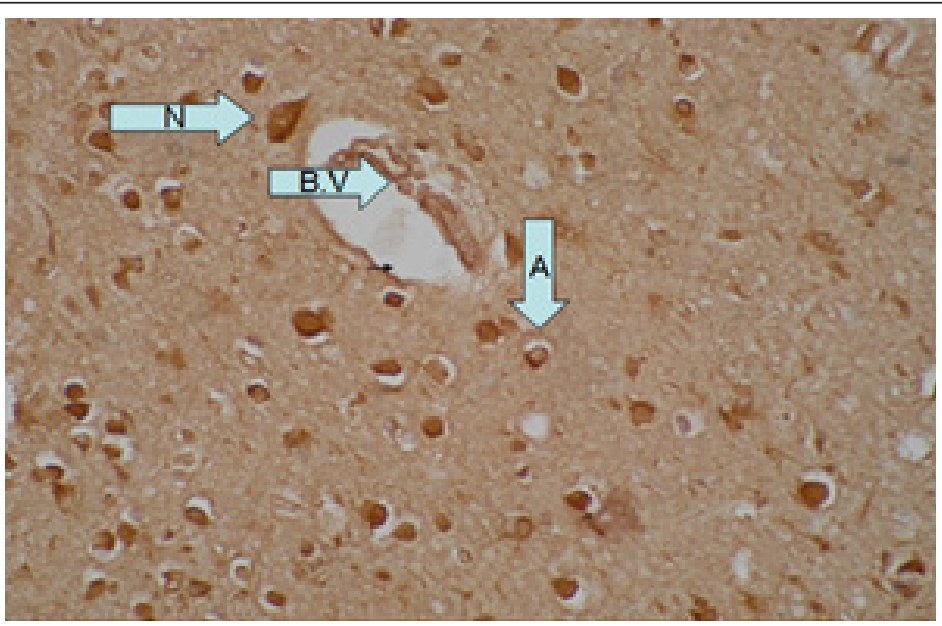

Figure 2: Shows strong expression of iNOS in brain hemorrhage case (+3 staining intensity in $A, N$ and B.V).

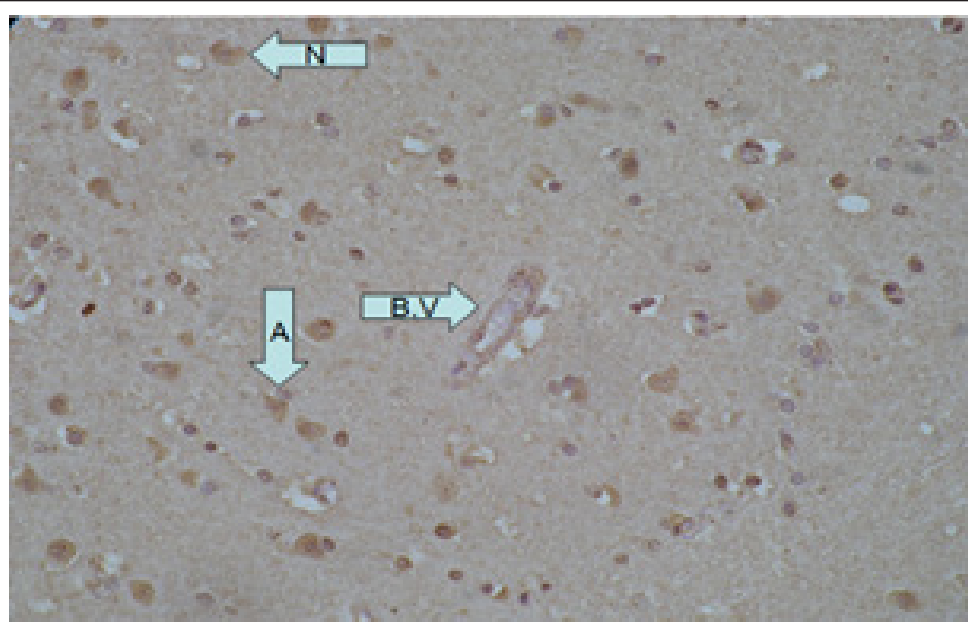

Figure 3: Shows moderate expression of iNOS in congestive brain case (+2 staining intensity in A, N and B.V). 
Jet kind demonstrated that tumors derived from human colon Adenocarcinoma cell engineered to generate NO continuously [17]. They also descended that the exact of iNOS immunostaining was in related to progression increase in tumor [18]. Moreover, the role of iNOS is controversial, some experimental showed the beneficial role of enzyme, but in other contradicting findings were reported. Hence for the work on the enzyme is needed on the all mentioned aspects to know it actual role. Then we can use its expression as a biological marker associate with brain injury cases in forensic medicine.

\section{Methodology}

A total of 80 cases (males 62, females 18) were included in the study. Among the total 21 (26.3\%) cases of TBI, 27 (33.8\%) cases of hemorrhage and 32 (39.9) cases of congestion. These cases were selected from the archives of Forensic Medicine Center at Jordan University of Science and Technology (JUST) from 2000-2008. The subject who were not diagnosed using neuroprotective drugs or as brain injury cases or had multiple reasons for death were excluded. The age range of the study population was 2 to 90 years. These were $37(46.2 \%)$ below the age of 30 and $43(53.8 \%)$ above the age of 30.23 cases of normal brain having same age grouped were also investigated as a control group. The attendant of all the cases were informed about the aim of the study and gave written consents for the investigation in accordance with ethical guideline.

Tissue sample were obtained from cerebral cortex site of both control and study groups. Section from infected lung tissues known to express iNOS were processed as positive control. The paraffin tissue blocks were prepared, then cut on a freezing microtome into $5 \mu \mathrm{m}$ transverse sections and processed free-floating to make slide as per standard laboratory procedure. The slides were prepared and immersed for 05 minute first in acetone and then in vecta bond reagent according to instruction of vector laboratories USA. After that slides were washed with D.W, followed by drying in oven at $60^{\circ} \mathrm{C}$ for 30 minutes. Immunohistochemical detections of iNOS was performed using commercially available monoclonal mouse antibody specific iNOS supplied by Santa Criuz biotechnology (SC651). iNOS Antibody was used with the dilutions of 1:60.

Immunohistochemical detections of iNOS was localized by using labeled streptavidin biotin LSAB kit (BIOGENIC), which consists of secondary biotinylated goat anti-mouse antibody and conjugated streptavidin Horse radish peroxidase followed by BIOGENIC MEDICAL DAB chromogen. Immunostaining was followed by counterstaining with Meyers hematoxylin. Parallel sections incubated with control serum (non-immunized serum) from Biogenic instead of the primary antibodies were used as negative controls. Then the slides were counterstained with hematoxylin for about 30 seconds. All slides were examined immunohistochemical evaluation by consultant pathologist. Scoring of iNOS expression was performed by subjective measure of intensity of staining as a measuring factor for the concentration of the antigen under studied.

Labeling Index (LI) was calculate from percentage of positive cells detected among the total cell under microscope and grading was performed as the criteria of PAN Jian-Wei system, According to this grading system, LI was used to classify positive cells into 4 grades: grade $0(\mathrm{LI}<5 \%)$; grade $1(\mathrm{LI}=5-25 \%)$; grade 2 ( $\mathrm{LI}=25-$ $50 \%$ ); grade 3 (LI $>50 \%$ ) 9. Statistical analysis was performed using the statistical software SPSS version 11.0.

\section{Result}

Brain's tissue samples of 23 normal subject and 80 cases was collected after surgery. iNOS was determined by immunohistochemistry with specified antibodies. The expression of the enzyme was scored in neurons, astrocytes and blood vessels. The scoring for expression was categorized as no staining (negative), mild, moderate and strong as per intensity of reaction related to the concentration of antigen. The result of our analysis is descried in the control group no iNOS staining in a neurons, astrocytes and blood vessels. Among study group (Table 1), iNOS staining reaction was more in neuron (58 cases), followed by astrocytes (51 cases) and blood vessels (45 cases). Negative staining was found more in blood vessels and less in neurons.

Table 1: Enzyme iNOS activity in the injured brain's constituents.

\begin{tabular}{|c|c|c|c|}
\hline Blood vessels & Astrocytes & Neurons & Staining result \\
\hline 35 & 29 & 22 & No stain \\
\hline 21 & 13 & 16 & Mild stain \\
\hline 9 & 13 & 1 & Moderate stain \\
\hline 15 & 25 & 41 & Strong stain \\
\hline
\end{tabular}

Table 2: Staining scores and type of brain injury.

\begin{tabular}{|c|c|c|c|}
\hline Type/Staining site & TBI (21) & Hemorrhage (27) & Congestion (32) \\
\hline Neurons & $18(85.7 \%)$ & $2(81.5 \%)$ & $18(56.3 \%)$ \\
\hline Astrocytes & $19(90.5 \%)$ & $19(70.4 \%)$ & $13(40.6 \%)$ \\
\hline Blood vessels & $16(76.2 \%)$ & $18(66.7 \%)$ & $11(34.4 \%)$ \\
\hline
\end{tabular}

(Table 2) Showed marked difference of iNOS activity in control and study group $(\mathrm{p}<0.05)$. Among TBI, only 3 cases were negative,
2 in astrocytes and 5 in blood vessels. Whereas in hemorrhage 5, 8 and 9 while in congestion 14,19,21 cases were observed negative 
in neurons, astrocytes and blood vessels respectively. Hence the iNOS activity scored more in TBI and hemorrhage cases and less in congestion cases. The enzyme showed no association in expression with respect to age, sex and brain weight.

\section{Discussion}

As iNOS found only under inflammatory condition, that is why we focused our study to the iNOS expression in brain injury cases. Our results highlighted that the changes occurred in the enzyme activity had no relation to age, sex and brain's weight. Besides, in normal human brain, the activity of enzyme was found NIL. Our findings are in consistent to the previous study who also found zero iNOS expression in normal colonic epithelium. We observed that iNOS activity in neurons was strong as compared to other sites in all types of brain injury cases. This is in line with other studies in which iNOS was expressed in epithelial cells, endothelial cells, macrophages, and neurons. The possible mechanism for the strong reaction may be that the action of $\mathrm{NO}$ often requires some other stimulation of target neurons before it can be effective. NO can also mediate the uptake and release of other neurotransmitters.

Moreover, NO blocks neurotransmitter receptors in brain injury cases. Second common staining was observed in astrocytes. Like ours, several other studies also showed that iNOS activity is localized in astrocytes especially reactive astrocytes. One study described that NO attenuates astrocytic glutamate uptake and induces brain injury via effects on astrocyte function. This may be the reason that we found $90.5 \%$ positive cases in TBI and $70.4 \%$ in hemorrhage. One more study, also found that the induction of iNOS occurs predominantly in reactive astrocytes, our data revealed that blood vessels were not as affected by iNOS activity as neurons and astrocytes. iNOS has been reported to influence local cerebral blood flow. This effect is reported to be mediated through cGMP- gated ion channels.

In addition, contrasting findings have been found even regarding the cellular localization of iNOS within the tumor samples. Taken together, these observations suggest a relevant role of iNOS and its product NO in the inflammatory diseases. Jen kin et al. [17] demonstrated that tumors derived from human colon adenocarcinoma cells engineered to generate NO continuously. They also described that the extent of iNOS immunostaining was in relation to progressive increase in tumor. Moreover, the role of iNOS is controversial; some experimental studies showed the beneficial role of enzyme, but in other contradicting findings were reported. Hence further work on the enzyme is needed on all the mentioned aspects to know its actual role. After then we can use its expression as a biological marker associated with brain injury cases in forensic medicine.

\section{Conclusion}

The activity of iNOS was shown to be significantly correlated with TBI and hemorrhage. Strong reactions were localized within neurons and astrocytes. This possibly explains the mediated effects of iNOS on brain blood vessels in terms of blood vessels dilatation to accommodate with the severity of brain injury cases.

\section{References}

1. Attwell D, Buchan AM, Charpak S, Lauritzen M, Macvicar BA, et al. (2010) Glial and neuronal control of brain blood. Nature 468: 232-243.

2. Lassmann H (1979) Basic mechanism of brain inflammation. Neural trans 50: 183-190.

3. McGur PL, McGur EG (1995) The inflammatory response system of brain. Inflammation for therapy of diseases. Brain Rev 21(2): 195-218.

4. Clerk RS, Multip NF, Kappa F (1998) Enhancer elements regulate cytokine of the human iNOS gene. J Biol chem 273: 15148-15156.

5. Guihot G, Guimbaud R, Bertraud V, Lambare BN, Couturier D, et al. (2004) iNOS activity in colon biopsies form inflammatory area. Amino acids 18(3): 229-237.

6. Cianchi F, Cortisim E, Fantappie 0, Messerini L, Schiavone N, et al. (2003) iNOS expression in human colorectal cancer. Am j of pathology 162(3): 793-801.

7. Simmon ML, Murph S (1992) Induction of nitric oxide synthase in glial cells. J Neurochmistry 59(3): 897-905.

8. Moshage H (1997) Nitric oxide determinations: much ado about NOthing? Chin chem 43(4): 553-556.

9. Pan JW, Zhan RY, Tong Y, Zhou YQ, Zhang M (2005) Expression of endothelial nitric oxide synthase and vascular endothelial growth factor in association with neovascularization in human primary astrocytoma. J Zhejian univ Sci 6(7): 693-698.

10. Moochhala S, Chhatwal VJ, Chan ST, Ngoi SS, Chia YW, et al. (1996) Nitric oxide synthase activity and expression in human colorectal cancer. Carcinogensis 17(5): 1171-1174.

11. Belvisi M, Barnes PJ Lakrin S, Yacoub M, Tadjkarimi S, et al. (1995) Nitric oxide synthase activity is elevated in inflammatory lung disease in humans. Eur J Pharm 283(1-3): 255-258.

12. Garthwaite J, Boulton $\mathrm{Cl}$ (1995) Nitric oxide signaling in the central nervous system. Ann Rev Physiol 57: 683-706.

13. Cianchi F, Cortesini C, Fantappie O, Messerini L, Schiavone N, et al. (2003) Inducible nitric oxide synthase expression in human colorectal cancer. Am j Pathology 162(3): 793-801.

14. Mason RB, Pluta RM, Walbridge S, Wink DA, Oldfield EH, et al. (2000) Production of reactive oxygen species after reperfusion in vitro and in vivo: Protective effect of nitrid oxide. J Neurosurg 93(1): 99-107.

15. Sakamoto KI, Fujisawa H, Koizumi H, Tsuchida E, Ito H, et al. (1997) Effects of mild hypothermia on nitric oxide synthesis following contusion trauma in the rat. J Neurotrauma 14(5): 349-353.

16. Ropponen KM, Kellokoski JK, Lipponen PK, Eskelinen MJ, Alanne L, et al. (2000) Expression of iNOS in colorectal cancer and its association with prognosis. Scand. J Gasteroenterol 35(11): 1204-1211.

17. Jenkins DC, Chorles JG, Thamson LL, Moss DW, Holmes LS, et al. (1995) Role of nitric oxide in tumor growth. Proc Natl Acad Sci USA 92(10): 4392-4396.

18. Puram SV, Yeung CM, Asl AJ, Lin C, Iglesia N, et al. (2012) STAT3iNOS Signaling Mediates EGFRvIII-Induced Glial Proliferation and Transformation. Neurosci 32(23): 7806-7818. 